Equations aux dérivées partielles

\title{
Localization of solutions for nonlinear elliptic problems with critical growth
}

\author{
Rejeb Hadiji Riccardo Molle Donato Passaseo Habib Yazidi
}

\begin{abstract}
We study the existence and the multiplicity of solutions for the problem $-\operatorname{div}(p(x) \nabla u)=$ $u^{2^{*}-1}+\lambda u, u>0$ in $\Omega$ and $u=0$ on $\partial \Omega$, when the set of the minimizers for the weight $p$ has multiple connected component. We study also the case where this set has one connected component and has complex topology.
\end{abstract}

\section{Localisation des solutions pour un problème elliptique avec exposant critique de Sobolev}

Résumé. On étudie l'existence et la multiplicité de solutions du problème $-\operatorname{div}(p(x) \nabla u)=u^{2^{*}-1}+$ $\lambda u, u>0$ dans $\Omega$ et $u=0$ sur $\partial \Omega$ dans le cas où l'ensemble de minima de $p$ admet plusieurs composantes connexes. On s'intéresse également au cas où cet ensemble possède une seule composante connexe et une topologie complexe.

Version française abrégée. On considère le problème suivant

$$
\left(P_{\lambda}\right) \quad\left\{\begin{aligned}
-\operatorname{div}(p(x) \nabla u) & =u^{2^{*}-1}+\lambda u, u>0 & & \operatorname{dans} \Omega \\
u & =0 & & \text { sur } \partial \Omega
\end{aligned}\right.
$$

où $\Omega$ est un domaine borné de $\mathbb{R}^{n}, n \geq 3,2^{*}=\frac{2 n}{n-2}, \lambda \in \mathbb{R}$ et $p: \bar{\Omega} \rightarrow \mathbb{R}$ une fonction positive dans $H^{1}(\Omega) \cap C(\bar{\Omega})$.

Soit $V(\Omega)=\left\{u \in H_{0}^{1}(\Omega): \int_{\Omega}|u(x)|^{2^{*}} d x=1\right\}$, on définit

$$
Q_{\lambda}(u)=\int_{\Omega} p(x)|\nabla u(x)|^{2} d x-\lambda \int_{\Omega}|u(x)|^{2} d x .
$$

On considère $S_{\lambda}(p)=\inf \left\{Q_{\lambda}(u): u \in V(\Omega)\right\}$. Soit $\lambda_{1}$ la première valeur propre de l'opérateur $-\operatorname{div}(p(x) \nabla \cdot)$ dans $H_{0}^{1}(\Omega)$. On sait (voir [7]) qu'il existe $0 \leq \lambda^{*}<\lambda_{1}$ tels que pour $\lambda<\lambda^{*}$, $S_{\lambda}(p)$ n'est pas atteint et pour $\lambda>\lambda^{*}, S_{\lambda}(p)$ est atteint. On suppose qu'il existe $\bar{x} \in \Omega$ vérifiant

$$
p(x) \leq \inf _{\Omega} p+c|x-\bar{x}|^{\alpha} \quad \forall x \in B(\bar{x}, r)
$$

avec $c>0, r>0$ et $\alpha>0$. Lorsque $n \geq 4$ et $\alpha>2$ on a $\lambda^{*}=0$, par contre si $n=3$ ou $0<\alpha \leq 2$ la situation est plus compliquée (voir [7]).

Dans cette Note, on s'intéresse au cas où $n \geq 4$ et $\alpha>2$ (voir [8] pour les autres cas).

On utilise la fonction dite "barycentre" $\beta: V(\Omega) \rightarrow \mathbb{R}^{n}$ définie par

$$
\beta(u)=\int_{\Omega} x|u(x)|^{2^{*}} d x \quad \forall u \in V(\Omega) .
$$

Nos résultats principaux sont les suivants: 
Théorème 1 Soit $\Omega$ un domaine borné de $\mathbb{R}^{n}, n \geq 4$. On suppose qu'il existe $\bar{x} \in \Omega$ et un ouvert $A \subseteq \mathbb{R}^{n}$ tels que $\bar{x} \in A$ et si $\bar{\Omega} \cap \partial A \neq \emptyset$ on $a \inf _{\bar{\Omega} \cap \partial A} p>\inf _{\Omega} p$. Alors, il existe $\left.\left.\bar{\lambda}_{A} \in\right] 0, \lambda_{1}\right]$ tel que, pour tout $\lambda \in] 0, \bar{\lambda}_{A}$ [, le problème $\left(P_{\lambda}\right)$ admet au moins une solution $u_{\lambda, A}$ qui vérifie $\beta\left(\frac{u_{\lambda, A}}{\left\|u_{\lambda, A}\right\|_{L^{2}}}\right) \in A$ et $Q_{\lambda}\left(\frac{u_{\lambda, A}}{\left\|u_{\lambda, A}\right\|_{L^{2 *}}}\right)=S_{\lambda}(p, A)(\operatorname{voir}(4))$.

Corollaire 1 On suppose qu'il existe $k$ ouverts disjoints dans $\mathbb{R}^{n}, A_{1}, \ldots, A_{k}$, et $k$ points $\bar{x}_{1}, \ldots, \bar{x}_{k}$ dans $\Omega$ vérifiant les mêmes conditions que $A$ et $\bar{x}$ du Théorème 1 . Alors, il existe $\left.\bar{\lambda} \in] 0, \lambda_{1}\right]$ tel que, pour tout $\left.\lambda \in\right] 0, \bar{\lambda}\left[\right.$, le problème $\left(P_{\lambda}\right)$ admet au moins $k$ solutions distinctes $u_{\lambda, A_{1}}, \ldots, u_{\lambda, A_{k}}$ vérifiant, pour $i=1, \ldots, k, \beta\left(\frac{u_{\lambda, A_{i}}}{\left\|u_{\lambda, A_{i}}\right\|_{L^{2}}}\right) \in A_{i}$ et $Q_{\lambda}\left(\frac{u_{\lambda, A_{i}}}{\left\|u_{\lambda, A_{i}}\right\|_{L^{2^{*}}}}\right)=S_{\lambda}\left(p, A_{i}\right)$ $(\operatorname{voir}(4))$.

Théorème 2 Soit $\Omega$ un ouvert borné dans $\mathbb{R}^{n}, n \geq 4$. On suppose qu'il existe un ouvert $A$ dans $\mathbb{R}^{n}$ et un fermé $K \subseteq \Omega \cap A$ telles que, pour tout $\bar{x} \in K$, les conditions du Théorème 1 sont vérifiées pour des constantes convenables $c>0, r>0$ et $\alpha>2$ (c, $r, \alpha$ sont indépendentes de $\bar{x})$. Alors, il existe $\bar{\lambda}>0$ tel que, pour tout $\lambda \in] 0, \bar{\lambda}\left[\right.$, le problème $\left(P_{\lambda}\right)$ admet au moins cat $_{A} K$ solutions distinctes, où cat ${ }_{A} K$ représente le Ljusternik-Schnirelman catégorie de $K$ dans $A$.

De plus, lorsque $\lambda$ tend vers 0 , chaque solution se concentre en un point minimum de $p$.

Let us consider the following problem

$$
\left(P_{\lambda}\right) \quad\left\{\begin{aligned}
-\operatorname{div}(p(x) \nabla u) & =u^{2^{*}-1}+\lambda u, u>0 & & \text { in } \Omega \\
u & =0 & & \text { on } \partial \Omega
\end{aligned}\right.
$$

where $\Omega$ is a bounded domain of $R^{n}, n \geq 3,2^{*}=\frac{2 n}{n-2}, \lambda \in \mathbb{R}$ and $p: \bar{\Omega} \rightarrow \mathbb{R}$ is a positive function in $H^{1}(\Omega) \cap \mathcal{C}(\bar{\Omega})$.

Let us denote by $\lambda_{1}$ the first eigenvalue of the operator $-\operatorname{div}(p(x) \nabla \cdot)$ in $H_{0}^{1}(\Omega)$. Consider the set $V(\Omega)=\left\{u \in H_{0}^{1}(\Omega): \int_{\Omega}|u(x)|^{2^{*}} d x=1\right\}$ and define the functional

$$
Q_{\lambda}(u)=\int_{\Omega} p(x)|\nabla u(x)|^{2} d x-\lambda \int_{\Omega}|u(x)|^{2} d x \quad \forall u \in V(\Omega) .
$$

For every $\lambda \in \mathbb{R}$, let us set

$$
S_{\lambda}(p)=\inf \left\{Q_{\lambda}(u): u \in V(\Omega)\right\}
$$

Notice that $S_{\lambda}(p)$ is a non increasing continuous function with respect to $\lambda$; the definition of $\lambda_{1}$ implies $S_{\lambda_{1}}(p)=0$; moreover, well known concentration arguments show that $S_{\lambda}(p)=S \inf _{\Omega} p$ $\forall \lambda \leq 0$, where $S$ denotes the best Sobolev constant, i.e. $S=\inf \left\{\int_{\Omega}|\nabla u|^{2} d x: u \in V(\Omega)\right\}^{\Omega}$. The infimum $S_{\lambda}(p)$ is not achieved for any $\lambda<\lambda^{*}$, where $\lambda^{*}=\max \left\{\lambda \in \mathbb{R}: S_{\lambda}(p)=S \inf _{\Omega} p\right\}$; on the contrary, $S_{\lambda}(p)$ is achieved for every $\lambda>\lambda^{*}$ and, if $\left.\lambda \in\right] \lambda^{*}, \lambda_{1}$ [, the minimizing functions give rise to solutions of problem $\left(P_{\lambda}\right)$ (taking into account the homogeneity of the nonlinear term).

The number $\lambda^{*}$ depends on the behaviour of the function $p(x)$ near its minimum points (see $[7,15])$. In particular, if there exists $\bar{x} \in \Omega$ such that $p(x) \leq \inf _{\Omega} p+c|x-\bar{x}|^{\alpha} \forall x \in B(\bar{x}, r)$ for suitable positive constants $c$ and $r$, then $\lambda^{*}=0$ when $n \geq 4$ and $\alpha>2$ (while the situation is more complex if $n=3$ or $0<\alpha \leq 2$ ). 
In this Note, for the sake of simplicity, we consider only this case (see [8] for the other cases); for $\lambda>0$ small enough, we obtain solutions which are localized near a prescribed region of minimizers for $p$; as $\lambda$ tends to 0 , these solutions concentrate as Dirac masses near minimum points of $p(x)$ and, after rescaling, converge to minimizing functions for the best Sobolev constant (see [14]); moreover, we exploit this concentration phenomenon in order to relate the number of solutions to the geometrical properties of the set of the minimizers of $p$.

When $p=1$ and $\lambda \rightarrow 0^{+}$problem $\left(P_{\lambda}\right)$ was studied in [12] and [13]. The effect of weights in front of the nonlinearity has considered by several authors see for example [1], [9] and the references therein. Note that, in subcritical case, Musso and Passaseo obtained the existence and multiplicity of nontrivial solutions for problems having asymptotically singular weights in divergence form operators see [11].

We shall use the barycenter function $\beta: V(\Omega) \rightarrow \mathbb{R}^{n}$ defined by

$$
\beta(u)=\int_{\Omega} x|u(x)|^{2^{*}} d x \quad \forall u \in V(\Omega)
$$

Lemma 1 Assume that $n \geq 4$ and that there exist $\bar{x} \in \Omega$ and $\alpha>2$ such that

$$
p(x) \leq \inf _{\Omega} p+c|x-\bar{x}|^{\alpha} \quad \forall x \in B(\bar{x}, r)
$$

for suitable positive constants $c$ and $r$. Moreover, assume that there exists an open set $A$ in $\mathbb{R}^{n}$ such that $\bar{x} \in A$ and, if $\bar{\Omega} \cap \partial A \neq \emptyset, \inf _{\bar{\Omega} \cap \partial A} p>\inf _{\Omega} p$. Then, there exists $\tilde{\lambda}_{A}>0$ such that the infimum

$$
S_{\lambda}(p, A)=\inf \left\{Q_{\lambda}(u): u \in V(\Omega), \beta(u) \in A\right\}
$$

is achieved for every $\lambda \in] 0, \tilde{\lambda}_{A}[$.

Proof. Notice that $\{u \in V(\Omega): \beta(u) \in A\} \neq \emptyset$ (because $\bar{x} \in \Omega \cap A$ ) and $S_{\lambda}(p, A)$ is continuous and non increasing with respect to $\lambda$. Moreover (concentrating near $\bar{x}$ the minimizing functions for the Sobolev constant and arguing as in $[5,7,15])$ one can show that $S_{\lambda}(p, A)=S \inf _{\Omega} p \forall \lambda \leq 0$ while (since $n \geq 4$ and $\alpha>2) S_{\lambda}(p, A)<S \inf _{\Omega} p \forall \lambda>0$.

Now, if $\{u \in V(\Omega): \beta(u) \in \partial A\} \neq \emptyset$, let us consider the infimum

$$
S_{\lambda}(p, \partial A)=\inf \left\{Q_{\lambda}(u): u \in V(\Omega), \beta(u) \in \partial A\right\} .
$$

It is clear that also $S_{\lambda}(p, \partial A)$ is continuous and non increasing with respect to $\lambda$.

Let us prove that $S_{0}(p, \partial A)>S \inf _{\Omega} p$. In fact, it is clear that $S_{0}(p, \partial A) \geq S \inf _{\Omega} p$. Arguing by contradiction, assume that the equality holds and consider a minimizing sequence $\left(u_{i}\right)_{i \in \mathbb{N}}$, i.e. $u_{i} \in V(\Omega), \beta\left(u_{i}\right) \in \partial A \forall i \in \mathbb{N}$ and $\lim _{i \rightarrow+\infty} \int_{\Omega} p(x)\left|\nabla u_{i}(x)\right|^{2} d x=S \inf _{\Omega} p$. Since $\int_{\Omega} p(x)|\nabla u(x)|^{2} d x \geq \inf _{\Omega} p \int_{\Omega}|\nabla u(x)|^{2} d x$, it follows that $\lim _{i \rightarrow+\infty} \int_{\Omega}\left|\nabla u_{i}(x)\right|^{2} d x=S$. Therefore, there exists $x_{0} \in \bar{\Omega}$ such that (up to a subsequence) $\left|\nabla u_{i}\right|^{2} \rightarrow S \delta_{x_{0}}$ and $\left|u_{i}\right|^{2^{*}} \rightarrow \delta_{x_{0}}$, where $\delta_{x_{0}}$ denotes the Dirac mass in $x_{0}$, (see [10]).

If $\bar{\Omega} \cap \partial A=\emptyset$, we have a contradiction because $\beta\left(u_{i}\right) \in \partial A \forall i \in \mathbb{N}$ implies $x_{0} \in \partial A$. If $\bar{\Omega} \cap \partial A \neq \emptyset$, we have $x_{0} \in \bar{\Omega} \cap \partial A$ which (under our assumptions) implies $p\left(x_{0}\right)>\inf _{\Omega} p$. As a consequence, we obtain

$$
\lim _{i \rightarrow+\infty} \int_{\Omega} p(x)\left|\nabla u_{i}(x)\right|^{2} d x=S p\left(x_{0}\right)>S \inf _{\Omega} p
$$


which also gives a contradiction.

Therefore, we must have $S_{0}(p, \partial A)>S \inf _{\Omega} p$ and, since $S_{\lambda}(p, \partial A)$ depends continuously on $\lambda$, there exists $\tilde{\lambda}_{A}>0$ such that $\left.S_{\lambda}(p, \partial A)>S \inf _{\Omega} p \forall \lambda \in\right] 0, \tilde{\lambda}_{A}[$. Hence, we have

$$
\left.S_{\lambda}(p, A)<S \inf _{\Omega} p<S_{\lambda}(p, \partial A) \quad \forall \lambda \in\right] 0, \tilde{\lambda}_{A}[
$$

Now, we can prove that the infimum $S_{\lambda}(p, A)$ is achieved for every $\left.\lambda \in\right] 0, \tilde{\lambda}_{A}[$. In fact, using a well known result of Brezis and Lieb (see [4]) and arguing as in [5], from the inequality $S_{\lambda}(p, A)<S \inf _{\Omega} p$ one can infer that every minimizing sequence for $S_{\lambda}(p, A)$ is relatively compact in $H_{0}^{1}(\Omega)$ (for the strong $H_{0}^{1}$ topology). Thus, up to a subsequence, every minimizing sequence $\left(u_{i}\right)_{i \in \mathbb{N}}$ converges in $H_{0}^{1}(\Omega)$ to a function $\bar{u} \in V(\Omega)$ such that $Q_{\lambda}(\bar{u})=S_{\lambda}(p, A)$ and $\beta(\bar{u}) \in \bar{A}$.

In order to complete the proof, we need only to observe that $\beta(\bar{u}) \in A$. Indeed, if $\{u \in$ $V(\Omega): \beta(u) \in \partial A\}=\emptyset$, this fact is obvious; in the other case, it follows from the inequality $S_{\lambda}(p, A)<S_{\lambda}(p, \partial A)($ see $(6))$.

Theorem 1 Let $\Omega$ be a bounded domain of $\mathbb{R}^{n}, n \geq 4$, assume that there exists $\bar{x} \in \Omega$ and an open set $A \subseteq \mathbb{R}^{n}$ satisfying the same conditions as in Lemma 1 .

Then, there exists $\left.\left.\bar{\lambda}_{A} \in\right] 0, \lambda_{1}\right]$ such that, for every $\left.\lambda \in\right] 0, \bar{\lambda}_{A}\left[\right.$, problem $\left(P_{\lambda}\right)$ has at least one solution $u_{\lambda, A}$ such that $\beta\left(\frac{u_{\lambda, A}}{\left\|u_{\lambda, A}\right\|_{L^{2}}}\right) \in A$ and $Q_{\lambda}\left(\frac{u_{\lambda, A}}{\left\|u_{\lambda, A}\right\|_{L^{2}}}\right)=S_{\lambda}(p, A)$ (see (4)).

Proof. Let us set $\bar{\lambda}_{A}=\min \left\{\tilde{\lambda}_{A}, \lambda_{1}\right\}$ (see Lemma 1). Thus, we have $\left.S_{\lambda}(p, A)>0 \forall \lambda \in\right] 0, \bar{\lambda}_{A}[$. Lemma 1 implies that there exixts a minimizing function $\bar{u}_{\lambda, A}$ for the infimum $S_{\lambda}(p, A)$; clearly, we can assume $\bar{u}_{\lambda, A} \geq 0$ (otherwise we replace $\bar{u}_{\lambda, A}$ by $\left|\bar{u}_{\lambda, A}\right|$ ). Since $\{u \in V(\Omega): \beta(u) \in A\}$ is an open subset of $V(\Omega)$, it follows that $\bar{u}_{\lambda, A}$ is a critical point for the functional $Q_{\lambda}$ constrained on $V(\Omega)$. Hence, taking into account that $S_{\lambda}(p, A)>0$, one can easily verify that $u_{\lambda, A}=$ $\left[S_{\lambda}(p, A)\right]^{\frac{n-2}{4}} \bar{u}_{\lambda, A}$ solves problem $\left(P_{\lambda}\right)$ and satisfies the desired properties.

The following corollary shows that Lemma 1 may be also used to obtain multiplicity of solutions when the set of the minimizers for $p(x)$ consists of several connected components.

Corollary 1 Let $\Omega$ be a bounded domain of $\mathbb{R}^{n}, n \geq 4$, and assume that there exist $k$ pairwise disjoint open sets in $\mathbb{R}^{n}, A_{1}, \ldots, A_{k}$, and $k$ points $\bar{x}_{1}, \ldots, \bar{x}_{k}$ in $\Omega$ such that, for $i=1, \ldots, k, \bar{x}_{i}$ and $A_{i}$ satisfy the same conditions as $\bar{x}$ and $A$ in Lemma 1.

Then, there exists $\left.\bar{\lambda} \in] 0, \lambda_{1}\right]$ such that, for every $\left.\lambda \in\right] 0, \bar{\lambda}\left[\right.$, problem $\left(P_{\lambda}\right)$ has at least $k$ distinct solutions $u_{\lambda, A_{1}}, \ldots, u_{\lambda, A_{k}}$ satisfying, for $i=1, \ldots, k, \beta\left(\frac{u_{\lambda, A_{i}}}{\left\|u_{\lambda, A_{i}}\right\|_{L^{2^{*}}}}\right) \in A_{i}$ and $Q_{\lambda}\left(\frac{u_{\lambda, A_{i}}}{\left\|u_{\lambda, A_{i}}\right\|_{L^{2}}}\right)=$ $S_{\lambda}\left(p, A_{i}\right)($ see (4)).

The proof is a direct consequence of Lemma 1 and Theorem 1.

Remark 1 When $\lambda$ tends to 0 , for each $i=1, \ldots, k$ the solution $u_{\lambda, A_{i}}$ given by Corollary 1 tends to concentrate (up to a subsequence) as a Dirac mass near a point $x_{i} \in A_{i}$ such that $p\left(x_{i}\right)=\inf _{\Omega} p$, and, after rescaling, converges to a minimizing function for the best Sobolev constant. This property follows easily taking into account that $\lim _{\lambda \rightarrow 0} S_{\lambda}\left(p, A_{i}\right)=S \inf _{\Omega} p$; in fact, as a consequence, we have

$$
\lim _{\lambda \rightarrow 0}\left\|u_{\lambda, A_{i}}\right\|_{L^{2^{*}}}^{-2} \int_{\Omega} p(x)\left|\nabla u_{\lambda, A_{i}}(x)\right|^{2} d x=S \inf _{\Omega} p
$$


which also implies

$$
\lim _{\lambda \rightarrow 0}\left\|u_{\lambda, A_{i}}\right\|_{L^{2^{*}}}^{-2} \int_{\Omega}\left|\nabla u_{\lambda, A_{i}}(x)\right|^{2} d x=S .
$$

Corollary 1 guarantees the existence of multiple solutions of Problem $\left(P_{\lambda}\right)$ in a case where the set of the minimizers for the weight $p(x)$ has multiple connected components; however, notice that we can have multiple solutions even if this set has only one connected component but it has complex topology. For example, the following result holds.

Theorem 2 Let $\Omega$ be a bounded domain of $\mathbb{R}^{n}, n \geq 4$, and assume that there exist an open set $A$ in $\mathbb{R}^{n}$ and a closed set $K \subseteq \Omega \cap A$ such that, for every $\bar{x} \in K$, the conditions required in Lemma 1 are satisfied for suitable constants $c>0, r>0$ and $\alpha>2(c, r, \alpha$ are independent of $\bar{x})$.

Then, there exists $\bar{\lambda}>0$ such that, for every $\lambda \in] 0, \bar{\lambda}\left[\right.$, problem $\left(P_{\lambda}\right)$ has at least $\operatorname{cat}_{A} K$ distinct solutions, where $\operatorname{cat}_{A} K$ denotes the Ljusternik-Schnirelman category of $K$ in $A$, i.e. the smallest integer $h$ for which there exist $h$ closed subsets of $A$, we call $K_{1}, \ldots, K_{h}$, which are contractible in $A$ and such that $K \subseteq \cup_{i=1}^{h} K_{i}$.

Moreover, as $\lambda$ tends to 0 , these solutions tend to concentrate as Dirac masses near points of $A$, which minimize the function $p(x)$, and, after rescaling, converge to minimizing functions for the best Sobolev constant.

Proof. Since $n \geq 4$ and $\alpha>2$, we have $S_{\lambda}(p, A)<S \inf _{\Omega} p \forall \lambda>0$ and if $\{u \in V(\Omega): \beta(u) \in$ $\partial A\} \neq \emptyset$, using Lemma 1 there exists $\bar{\lambda}>0$ such that

$$
\inf \left\{Q_{\lambda}(u): u \in V(\Omega), \beta(u) \in \partial A\right\}>S \inf _{\Omega} p \quad \forall \lambda<\bar{\lambda} .
$$

The concentration arguments used in $[3,5,7,15]$ show that, for every $\lambda>0$, one can find a nonnegative radial function $\varphi_{\lambda} \in H_{0}^{1}(B(0, r))$ such that, if we set $\varphi_{\bar{x}, \lambda}(x)=\varphi_{\lambda}(x-\bar{x}) \forall \bar{x} \in K$ $\left(\varphi_{\bar{x}, \lambda}(x)=0\right.$ for $\left.x \notin B(\bar{x}, r)\right)$ and consider the set $\Phi_{K, \lambda}=\left\{\varphi_{\bar{x}, \lambda}: \bar{x} \in K\right\}$, then $\Phi_{K, \lambda} \subseteq V(\Omega)$ and

$$
\sup \left\{Q_{\lambda}(u): u \in \Phi_{K, \lambda}\right\}<S \inf _{\Omega} p .
$$

Arguing as in $[5,7,15]$ and taking into account (7) and (8), one can show that the sublevel

$$
\left\{u \in V(\Omega): \beta(u) \in A, Q_{\lambda}(u) \leq \sup _{\Phi_{K, \lambda}} Q_{\lambda}\right\}
$$

is compact in $H_{0}^{1}(\Omega)$ for every $\left.\lambda \in\right] 0, \bar{\lambda}$.

Therefore, if we denote by $\tilde{k}$ the Ljusternik-Schnirelman category of $\Phi_{K, \lambda}$ in $\{u \in V(\Omega)$ : $\beta(u) \in A, u \geq 0$ in $\Omega\}$, general results of critical points theory guarantee that in this sublevel there exist at least $\tilde{k}$ critical points for the functional $Q_{\lambda}$ constrained on $V(\Omega)$, which give rise to $\tilde{k}$ distinct solutions of $\left(P_{\lambda}\right)$.

Now, notice that $\tilde{k} \geq \operatorname{cat}_{A} K$; in fact, if $H_{1}, \ldots, H_{h}$ are $h$ closed subsets contractible in $\{u \in V(\Omega): \beta(u) \in A, u \geq 0$ in $\Omega\}$ and such that $\Phi_{K, \lambda} \subseteq \cup_{i=1}^{h} H_{i}$, then the sets $K_{1}, \ldots, K_{h}$ defined by $K_{i}=\left\{\bar{x} \in K: \Phi_{\bar{x}, \lambda} \in H_{i}\right\}$, for $i=1, \ldots, h$, are closed subsets of $A$, are contractible in $A$ (as one can easily verify by using the barycenter function $\beta$ ), and $K=\cup_{i=1}^{h} K_{i}$.

Finally, let us remark that, as $\lambda$ tends to 0 , the asymptotic behaviour of the solutions follows easily taking into account that

$$
\lim _{\lambda \rightarrow 0} S_{\lambda}(p, A)=\lim _{\lambda \rightarrow 0} \sup _{\Phi_{K, \lambda}} Q_{\lambda}=S \inf _{\Omega} p .
$$

(arguing as in Remark 1) 
Remark 2 The solutions obtained in Corollary 1 correspond to $k$ local minimizers for the corresponding energy functional. Therefore, it is natural to expect that one could apply the mountain pass Theorem (see [2]) to find other solutions, corresponding to higher critical values. But new difficulties arise, due to the presence of the weight $p(x)$; in fact, the higher critical values of $p(x)$ produce new energy levels where the Palais-Smale compactness condition is not satisfied; so this condition may fail just at the mountain pass level.

However, under the assumptions required in Corollary 1, it is possible to find many higher energy solutions using a new approach (see [8]); in fact, for every subset $\left\{i_{1}, \ldots, i_{r}\right\}$ of $\{1, \ldots, k\}$, (by minimizing on a suitable manifold of codimension $r$ ) we construct a solution of $\left(P_{\lambda}\right)$ which is the sum of $r$ positive functions $u_{\lambda, i_{1}}, \ldots, u_{\lambda, i_{r}}$ that, for $\lambda>0$ small enough, are localized, respectively, near the prescribed regions $A_{i_{1}}, \ldots, A_{i_{r}}$. Hence, for every $r=1, \ldots, k$, we obtain

on the whole $\left(\begin{array}{l}k \\ r\end{array}\right)$ distinct $r$-peak solutions. Also in the case described in Theorem 2 it is natural to expect the existence of a higher energy solution when the set $K$ is not contractible in $A$ (i.e. cat $_{A} K \geq 2$ ). In this case (see [8]) the region where the weight $p(x)$ is large may play the same role as a hole in the domain (as in $[6,12]$ ); in general, the weight $p(x)$ may have the same effect as the domain shape on the existence and the multiplicity of solutions.

Finally, let us point out that the results presented in this Note may be also explained in terms of bifurcation with respect to the parameter $\lambda$. It is well known that a branch of positive solutions bifurcate from the first eigenvalue $\lambda_{1}$; some examples (see [8]) suggest that the existence of multiple solutions, given by Corollary 1 and Theorem 2, corresponds to secondary bifurcations from this branch of positive solutions.

\section{References}

[1] S. Alama - G. Tarantello,, Elliptic Problems with Nonlinearities Indefinite in sign, J. funct. Anal.141, (1996), 159-214.

[2] A. Ambrosetti - P. Rabinowitz, Dual variational methods in critical point theory and applications, J. Functional Analysis 14, (1973), 349-381.

[3] H. Brezis, Elliptic equations with limiting Sobolev exponents - the impact of topology, Comm. Pure Appl. Math. 39 , (1986), S17-S39.

[4] H. Brezis - E. Lieb, A relation between pointwise convergence of functions and convergence of functionals, Proc. A.M.S. 88, (1983), no. 3, 486-490.

[5] H. Brezis - L. Nirenberg, Positive solutions of nonlinear elliptic equations involving critical Sobolev exponents, Comm. Pure Appl. Math. 36, (1983), no. 4, 437-477.

[6] J.M. Coron, Topologie et cas limite des injections de Sobolev, C. R. Acad. Sci. Paris Sér. I Math. 299, (1984), 209-212.

[7] R. Hadiji - H. Yazidi, Problem with critical Sobolev exponent and with weight, to appear in Chinese Ann. of Maths. (Serie B).

[8] R. Hadiji - R. Molle - D. Passaseo - H. Yazidi, in preparation.

[9] Y. Li, On $-\Delta u=K(x) u^{5}$ in $\mathbb{R}^{3}$, Comm. Pure Appl. Math. 46, (1993), 303-340.

[10] P. L. Lions, The concentration-compactness principle in the calculus of variations, The limit case, part 1 and part 2, Rev. Mat. Iberoamericana, Vol 1, (1985), no. 1, 145-201 and (1985), no.2, 45-121.

[11] M. Musso - D. Passaseo, Multibump solutions for a class of nonlinear elliptic problems, Calc. Var. Partial Differential Equations 7, (1998), no. 1, 53-86.

[12] O. Rey, Sur un problème variationnel non compact: l'effet de petits trous dans le domaine, C. R. Acad. Sci. Paris Sér. I Math. 308, (1989), 349-352.

[13] O. Rey, The role of the Green's function in a nonlinear elliptic equation involving the critical Sobolev exponent, J. funct. Anal.89, (1990), no. 1, 1-52.

[14] G. Talenti, Best constants in Sobolev inequality, Ann. Mat. Pura Appl. 110, (1976), 353-372. 
[15] H. Yazidi, Etude de quelques EDP non linéaires sans compacité, PHD Thesis, Université Paris 12, (2006).

R.H. and H.Y.: UFR des Sciences et Technologie, CNRS UMR 8050, Université Paris 12 - Val-de-Marne, 61, avenue du Général de Gaulle, 94010 Créteil, France ;

R.M.: Dipartimento di Matematica Università di Roma "Tor Vergata" Via della Ricerca Scientifica, 1, 00133 Roma, Italy ;

D.P.: Dipartimento di Matematica "E. De Giorgi”, Università di Lecce, P.O. Box 193, 73100 Lecce, Italy. 